\title{
Acadêmicas de enfermagem na atuação com gestantes e as principais dúvidas: relato de experiência
}

\author{
Nursing students in their work with pregnant women and the main doubts: experience \\ report
}

Estudiantes de enfermería en la actuación con mujeres embarazadas y las principales dudas: informe de experiencia

Isabelle de Souza Januaria1*, Paula Fernandes de Souza ${ }^{1}$, Myllene Aparecida Leite de Souza ${ }^{1}$, Isabela Mie Takeshita ${ }^{1}$.

\section{RESUMO}

Objetivo: Relatar a experiência de acadêmicas de enfermagem do terceiro período ao promover rodas de conversa com gestantes. Relato de experiência: Estudo descritivo, relato de experiência, fruto de um projeto de educação em saúde voluntário. Nas rodas de conversa foram abordados temas de demanda e baseados na caderneta do Ministério da Saúde. Foram dois encontros, o primeiro contou com a presença de três gestantes e o segundo com cinco. Pontos valorizados nas conversas foram os cuidados com o neonato, a importância da escolha e uso de métodos contraceptivos, que apesar de serem fornecidos pelo SUS, eram pouco conhecidos. A experiência favoreceu um aprendizado consolidado, pois cada acadêmica precisou estudar e se preparar para conduzir as rodas de conversa, de modo a favorecer a comunicação horizontal. Considerações finais: As rodas de conversa durante a formação acadêmica favorecem aos discentes uma aproximação com a realidade, compreendendo as demandas da sociedade e a sua importância como meio de disseminação do conhecimento, permite melhor compreensão dos estudos realizados em sala de aula e desenvolvimento de habilidades e competências fundamentais ao enfermeiro. Palavras-chave: Educação em saúde, Pré-natal, Recém-nascido.

\begin{abstract}
Objective: To register an academic nursing experience in the third period and promote conversation circles with pregnant women. Experience report: Descriptive study, experience report, the result of a voluntary health education project. In the conversation circles, topics of demand and base were addressed in the Ministry of Health booklet. There were two meetings, the first with the presence of three pregnant women and the second with five. Points valued in the conversations were care for the newborn, an importance of choosing and using contraceptive methods, which despite being used by SUS, were little known. The experience favored consolidated learning, as each academic needs to study and prepare to conduct themselves as conversation circles, in order to favor horizontal communication. Final considerations: Health education applied to this methodology offers academics an experience in assisting professional development as a promoter and instructor in health, allowing to reach the most diverse audiences. The horizontality provided by the permission to break barriers as a hierarchy of communication, allows all participants the possibility to share their knowledge.
\end{abstract}

Keywords: Health education, Prenatal, Newborn.

\footnotetext{
${ }^{1}$ Faculdade Ciências Médicas de Minas Gerais (FCMMG), Belo Horizonte - MG.

*E-mail: isabelle.souza1996@hotmail.com
}

SUBMETIDO EM: 4/2020

ACEITO EM: 5/2020

PUBLICADO EM: 7/2020 


\section{RESUMEN}

Objetivo: Registrar una experiencia académica de enfermería en el tercer período y promover círculos de conversación con mujeres embarazadas. Informe de experiencia: estudio descriptivo, informe de experiencia, el resultado de un proyecto voluntario de educación sanitaria. En los círculos de conversación, los temas de demanda y base se abordaron en el folleto del Ministerio de Salud. Hubo dos reuniones, la primera con la presencia de tres mujeres embarazadas y la segunda con cinco. Los puntos valorados en las conversaciones fueron el cuidado del recién nacido, la importancia de elegir y utilizar métodos anticonceptivos, que a pesar de ser utilizados por el SUS, eran poco conocidos. La experiencia favoreció el aprendizaje consolidado, ya que cada académico necesita estudiar y prepararse para comportarse como círculos de conversación, a fin de favorecer la comunicación horizontal. Consideraciones finales: Los círculos de conversación durante la capacitación académica favorecen a los estudiantes a acercarse a la realidad, comprender las demandas de la sociedad y su importancia como un medio para difundir el conocimiento, lo que permite una mejor comprensión de los estudios realizados en el aula y el desarrollo de habilidades y competencias fundamentales. a la enfermera.

Palabras clave: Educación para la salud, Prenatal, Recién nacido.

\section{INTRODUÇÃO}

A gestação gera transformações de ordem física, psicológica e social, tais mudanças são influenciadas por diferentes fatores, como biológicos, socioeconômicos e culturais. Desta forma, a gestante deve receber acolhimento qualificado no centro de saúde onde está inserida, com práticas para evitar intercorrências e promover sua saúde durante a gestação (CASTRO JFL e LIMA PM, 2017).

Ansiedade, expectativa, medo e angústia são sentimentos comuns do período da gestacional e que são acompanhados de dúvidas relacionadas aos cuidados durante a gestação, no parto e pós-parto. Diante de tantas incertezas a participação das gestantes em rodas de conversa possibilita a troca de experiências com profissionais de saúde e outras mulheres gestantes, o que favorece um sentimento de confiança e ameniza seus anseios. Neste contexto, a educação em saúde se torna fundamental para uma gestação tranquila e saudável (FROTA MA, et al., 2020).

A mulher é essencial no processo de ensino em saúde frente a família, pois realiza o cuidado da saúde de seus entes, atua como multiplicadora de informações e de ações que possam levar ao bem-estar familiar, consequentemente, à melhora da qualidade de vida. A aquisição de hábitos e escolhas saudáveis proporcionam a mudança de comportamento, promoção e manutenção de saúde do indivíduo (BITTAR CML e PINHEIRO BC, 2017).

Existe estreita relação entre a promoção da saúde e a educação em saúde, pois elas se estabelecem por meio da participação da população, do seu modo de vida, crenças, valores, desejos, de suas necessidades, enfim, de sua subjetividade e intersubjetividade, considerando seu contexto sócio-políticocultural. Tal participação requer que o indivíduo se envolva, se comprometa e seja solidário. A construção coletiva, que parte do cotidiano, com decisões em conjunto, permite a troca de experiências, vivências, conhecimentos, que serão distintos, uns dos outros, porque as histórias de cada um foram diferentemente elaboradas (DAROS DZ, et al., 2010).

O enfermeiro pode desenvolver estas estratégias de promoção à saúde durante as orientações no prénatal ou na consulta de puericultura. Infelizmente estas estratégias são, por vezes, esquecidas durante o atendimento no setor hospitalar ou até no domicílio. Torna-se primordial o desenvolvimento de uma assistência e de atividades educativas, considerando as condições sociais, ambientais e as relações familiares, de modo que a mulher gestante e/ou puérpera seja tratada na sua individualidade e integralidade (ATAIDE MBC, et al., 2015; CARVALHO ATF, et al., 2017). O acadêmico que viver experiências práticas de educação em saúde durante a sua graduação, terá um melhor progresso, além de ser uma forma de desenvolver habilidades interpessoais que irão ampliar as possibilidades de promoção à saúde em diversos 
cenários. Esta ação é de fundamental importância, dado que o enfermeiro, ao longo de sua profissão, propaga o seu conhecimento e partilha da educação em saúde (BARBOSA AL, et al., 2016). Justifica-se a elaboração do artigo pela importância depromover a educação em saúde entre estudantes, pois esta experiência favoreceu ao acadêmico o estabelecimento de competências fundamentais para o seu cotidiano profissional, competências essas cognitivas, atitudinais e procedimentais.

Por meio da realização de rodas de conversas é possível compartilhar conhecimento e trocas de experiências com a população partindo sempre da necessidade das gestantes acolhidas por um Centro de Saúde de Belo Horizonte, MG. O objetivo deste artigo é relatar a experiência de acadêmicas de enfermagem ao promover rodas de conversa com gestantes com a finalidade de estimular a autonomia.

\section{RELATO DE EXPERIÊNCIA}

O trabalho tratou- se de um estudo descritivo do tipo relato de experiência de um projeto de educação em saúde, com características de projeto de extensão, realizado no primeiro semestre de 2018 em dois encontros. Foram selecionadas quatro acadêmicas do $3^{\circ}$ período de enfermagem, que deveriam ter disponibilidade para participar dos encontros nos horários previamente estabelecidos. Desta forma, voluntariamente, colaboraram na manutenção e continuidade do grupo de gestantes de um Centro de Saúde de Belo Horizonte, MG.

No semestre em curso (10 de 2018), apenas um grupo de estágio supervisionado passou na unidade, promovendo uma roda de conversa, como elas deveriam ser mensais, optou-se por convidar acadêmicas de outro período para manter os encontros. Os temas abordados nos foram definidos em rodas de conversa anteriores e em reunião com a enfermeira do Centro de Saúde.

A forma escolhida para abordar os temas foi definida em reunião entre as acadêmicas, bem como destacadas as necessidades mais agudas do público alvo. A sala de reuniões da unidade era o cenário das rodas de conversa, tiveram duração média de uma hora e meia e as acadêmicas ofereciam um café da manhã como atrativo.

Nos dois encontros participaram oito gestantes, com idades entre 20 e 35 anos, havendo entre as participantes primíparas e multíparas. Todas estavam realizando acompanhamento pré-natal no Centro de Saúde em questão. No primeiro encontro estiveram presentes três gestantes, apenas uma delas estava acompanhada pelo companheiro, as demais estavam acompanhadas por familiares, como filhos ou irmãos.

As acadêmicas estudaram o conteúdo e prepararam slides que foram projetados na televisão, o uso de muitas imagens apoiaram as explicações, aproximando as gestantes do conteúdo. Notou-se interesse por parte das gestantes para esclarecer dúvidas a respeito da alimentação adequada e conhecer as alternativas mais acessíveis em termos de preço e valor nutricional. Foi possível ainda, discutir mitos e verdades divulgados pela cultura popular sobre a qualidade do leite materno e o aleitamento materno exclusivo.

O estudo prévio foi importante para as acadêmicas, elas ainda não tinham contato com disciplinas como Saúde da Criança, Nutrição ou Saúde da Mulher, por isso, utilizaram suas experiências pessoais, embasadas na literatura, para se colocarem com maior segurança no encontro, bem como no esclarecimento de dúvidas.

Com intuito de aproximar o conteúdo proposto com a realidade vivenciada pelas participantes, houve por parte das acadêmicas, alguns relatos de experiências particulares relacionados aos períodos de cuidado praticados com irmãos mais novos, além da exposição da experiência vivenciada pelas mães. Estes acréscimos de conteúdo contribuíram para o incremento da horizontalidade entre os participantes do encontro.

Apesar de incentivos do governo e da mídia, ainda há pouco conhecimento a respeito da qualidade do leite materno, isso foi evidenciado quando uma das mães revelou dúvida sobre a suficiência do leite materno para alimentar seu filho. Outra temática que gerou questionamentos entre as mães foi a importância da alimentação saudável e quais os alimentos mais indicados para o período gestacional. 
Utilizando a própria experiência alimentar, além do estudo prévio do conteúdo proposto pelo guia alimentar recomendado pela OMS, as acadêmicas repassaram as gestantes as informações contidas no manual, além de compartilhar estratégias particulares utilizadas para a aquisição de produtos saudáveis.

Os métodos contraceptivos também foram abordados, para que as gestantes reflitam antecipadamente sobre planejamento familiar. Elas estavam interessadas sobre todos: pílula anticoncepcional, anticoncepcional injetável, dispositivo intrauterino (DIU), camisinha masculina e feminina e até métodos não disponíveis no Sistema Único de Saúde (SUS), como o adesivo hormonal. Duas mães desconheciam o DIU, seu mecanismo, local de inserção, além de desconhecer que a sua distribuição e implantação se dá por meio do SUS, sem custo direto para a usuária. As gestantes presentes relataram que possuem interesse em aderir a algum dos métodos apresentados e foram orientadas a agendar consulta após o parto para esta definição.

Neste cenário as acadêmicas já haviam pesquisado como ocorria a distribuição dos métodos contraceptivos via SUS, bem como do centro de saúde frequentado pelas gestantes. Para abordar este item foi reportado as participantes as experiências vividas pelas acadêmicas no que diz respeito a utilização de métodos contraceptivos e quais foram os aspectos que as fizeram decidir entre a variedade disponibilizada.

Com intuito de dar sequência nos temas abordados na primeira roda de conversa, o enfoque do segundo encontro abordou cuidados voltados ao pós-parto materno e com o recém-nascido. $O$ formato roda de conversa permitiu que as gestantes trocassem experiências sobre as gestações anteriores e se preparar mais para a atual.

Os temas abordados foram: cuidados com o recém-nascido e o pós-parto. O assunto que gerou mais dúvidas com relação ao recém-nascido foi a icterícia. As gestantes presentes mostraram aversão ao método de tratamento utilizado, a fototerapia, por considerarem um pouco agressivo para o bebê. Em virtude dos questionamentos a docente explicou todos os benefícios que a fototerapia proporciona para o bebê. Foi necessário a participação da docente neste tópico pois o aprofundamento das dúvidas requeria maior prática e informações concisas e as acadêmicas não possuíam experiência nesta abordagem específica.

Os métodos contraceptivos foram retomados no segundo encontro pelo interesse e desconhecimento das gestantes sobre a disponibilidade e gratuidade dos métodos pelo SUS, foi abordado ainda o tempo indicado para retorno da vida sexual.

Os parceiros e acompanhantes eram convidados a participar das rodas de conversa e recebiam orientações sobre o direito de acompanhar o parto e as práticas que não deveriam ocorrer, principalmente aquelas que se configuram como violência obstétrica, além de demonstrar as formas de apoio à mãe no período gestacional e puerperal, além dos direitos que lhes são garantidos por lei.

O formato de roda de conversa permitiu às acadêmicas e docente obter um retrato social das gestantes abrangidas pelo Centro de Saúde em questão. Foi possível notar a presença de primíparas, multíparas e jovens que não haviam planejado a gestação. Os encontros revelaram a importância da educação em saúde na garantia da equidade de acesso à saúde e à informação.

\section{DISCUSSÃO}

Os encontros realizados no formato de roda de conversa oferecem maior interação entre as gestantes e as acadêmicas, as participantesse sentem confortáveis diante da aproximação e a linguagem de fácil entendimento proporcionam maior clareza nas informações.

Os encontros destinados à educação em saúde são de suma importância para a saúde pública e possuem espaço destinado dentro da linha de Cuidado à Gestante, Puérpera e ao Recém-Nascido, pois, a partir da instrução da população se faz possível a promoção em saúde e emancipação dos indivíduos (AZEVEDO EVS, et al., 2019).

Contribuindo diretamente para o desenvolvimento da escuta qualificada e diálogo profícuo dos acadêmicos, que é de extrema importância para o exercício da futura profissão, visto que dentre as 
competências do enfermeiro espera-se que haja escuta ativa e qualificada para orientação dos serviços e atendimento as demandas e necessidades dos indivíduos inseridos em determinada população (DIAS RM, et al., 2019).

Para a nutrição adequada deve-se levar em consideração a condição socioeconômica das puérperas para uma orientação acessível para essa família, de forma que seja possível seguir as orientações e que não se torne apenas motivo de frustrações. Diante disso, notou-se a importância de relacionar conhecimentos adquiridos na disciplina nutrição aplicada à saúde e no guia de alimentação da população brasileira, correlacionando as principais formas de alimentação e dispor de opções com melhor custobenefício para que essas mulheres pudessem se alimentar melhor, visando a saúde fisiológica e nutricional materno-infantil, abordando também os alimentos que devem ser evitados (OMS, 2014).

Apesar das recentes campanhas e enfrentamentos, as práticas de violência obstétrica ainda continuam (ESTUMANO VKC, et al., 2017), de acordo com uma das gestantes, em uma outra gravidez houve indução de parto com aplicação de ocitocina para aumento das contrações, o que fez com que a gestante sentisse muita dor.

De acordo com as recomendações da OMS no atendimento ao parto normal a administração deverá ser oferecida a mulher somente em casos em que se faça real necessidade, por exemplo, "administração profilática de ocitocina no terceiro estágio do parto em mulheres com risco de hemorragia no pós-parto, ou que correm perigo em consequência da perda de até uma pequena quantidade de sangue", outros casos devem ser avaliados individualmente para que não haja sofrimento da puérpera por meio de práticas consideradas violentas à mulher (SANTANA AT, et al., 2019).

Os meios de ação dos métodos contraceptivos disponíveis ou não por meio do Sistema Único de Saúde (SUS) foram explicados para as puérperas a fim de que houvesse possibilidade de escolha a partir do conhecimento. O Dispositivo Intra-Uterino (DIU) foi o que causou maior interesse por parte das ouvintes, em contrapartida, poucas sabiam da disponibilidade do DIU de cobre pelo SUS e seus benefícios, o que evidencia os impactos positivos da realização e das rodas de conversa e planejamento familiar como parte integrante do Programa de Saúde da Família e Planejamento Familiar.

De acordo com a Norma Operacional da Assistência à Saúde do SUS de 2001, cabe ao enfermeiro realizar as orientações de planejamento familiar, não somente realizando consultas de enfermagem aos indivíduos que buscam pelo serviço com a demanda de planejar uma gravidez, mas também aos usuários que visam evitar uma gestação indesejada, além da realização da busca ativa (DIAS RM, et al., 2019).

Desta forma, o enfermeiro deve estar ciente do seu papel na comunidade onde atua e, além de conhecer as estatísticas e prevalências, deve realizar diagnóstico situacional em sua área de abrangência, a fim de realizar as intervenções necessárias e planejar a educação em saúde voltada para aquela realidade, para tanto, deve-se desenvolver tais competências durante a academia (MOREIRA KFA, et al., 2019).

Infere-se que as rodas de conversa durante a formação acadêmica favorecem aos discentes uma aproximação com a realidade, compreendendo as demandas da sociedade e a sua importância como meio de disseminação do conhecimento, permite melhor compreensão dos estudos realizados em sala de aula e desenvolvimento de habilidades e competências fundamentais ao enfermeiro.

Foi possível aplicar a roda de conversa como método de abordagem horizontal, uma vez que por meio deste diálogo é possível que as gestantes tenham suas dúvidas esclarecidas, além de possibilitar a troca de experiências e a criação da rede de apoio, já que os diálogos são abertos aos familiares, de forma a trabalhar a importância do papel ativo no auxílio à mulher no período gravídico e pós-gravídico.

\section{REFERÊNCIAS}

1. AZEVEDO EV, et al. Educação em saúde no empoderamento da gestante. Revista de Enfermagem da UFPE online, [SI]. 2019; 13(5): 1527-1531. 
2. ALENDE LP, et al. Rede de apoio social de puérperas na prática da amamentação. Escola Anna Nery Revista de Enfermagem. 19. 2. Rio de Janeiro, Redalyc, 2015.

3. BITTAR CML, PINHEIRO BD. Práticas de educação popular em saúde na atenção primária: uma revisão integrativa. Revista do Departamento de Educação Física e Saúde e do Mestrado em Promoção da Saúde da Universidade de Santa Cruz. 2017, 18(1): 77-82.

4. BARBOSA AL, et al. Concepções das práticas de educação em saúde no contexto da formação em Enfermagem. Rev. Rene. 2016;17(4): 545-52.

5. BOCK A, et al. Ações de educação em saúde na atenção primária a gestantes e puérperas: revisão integrativa. Journal of Nursing. 2019; 13(2).

6. CARVALHO ATF, et al. Educação em saúde no pré-natal: estratégia de grupo. Revista Congresso Nacional de Práticas Educativas. 2017; 1.

7. CARVALHO MS, et al. Educação em saúde durante o pré-natal com foco nos cuidados relacionados ao recémnascido. Rev Cadernos de Graduação. 2016; 3(3); 195-208.

8. CASTRO JFL e LIMA PDM. Orientações no pré-natal de baixo risco acerca do parto humanizado: um estudo comparativo entre duas Unidades de Saúde da Família. Rev. Brasileira de Pesquisa em Saúde. 2017; 19(2); 115123.

9. DAROS DZ, et al. Socialização de conhecimentos e experiências sobre o processo de nascimento e tecnologias do cuidado. Rev. Eletr. Enf., 2010; 12(2): 308-314.

10. DIAS RM, MONIZ MA. Nursing managerial aptitudes in the family health strategy: perceptions of nursing undergraduates. Rev Fun Care Online. 2019; 11(4): 1048-1052.

11. FROTA MA, et al. Mapeando a formação do enfermeiro no Brasil: desafios para atuação em cenários complexos e globalizados. Ciênc. saúde coletiva, Rio de Janeiro. 2020; 25(1): 25-35.

12. MINISTÉRIO DA SAÚDE (BR). Secretaria de Atenção à saúde. Guia alimentar para a população brasileira. Brasília; 2014.

13. SANTANA AT, et al. Atuação de enfermeiras residentes em obstetrícia na assistência ao parto. Rev. Bras. Saude Mater. Infantil. Recife. 2019; 19(1): 135-144.

14. MOREIRA KFA, et al. Aprendendo, ensinando e mapeando território: vivências de acadêmicos de enfermagem. Revista Eletrônica Acervo Saúde. 2019; 11(4).

15. ESTUMANO VKC, et al. Violência obstétrica no Brasil: casos cada vez mais frequentes. Revista Científica de Enfermagem. São Paulo. 2017; 7(19): 83-91. 